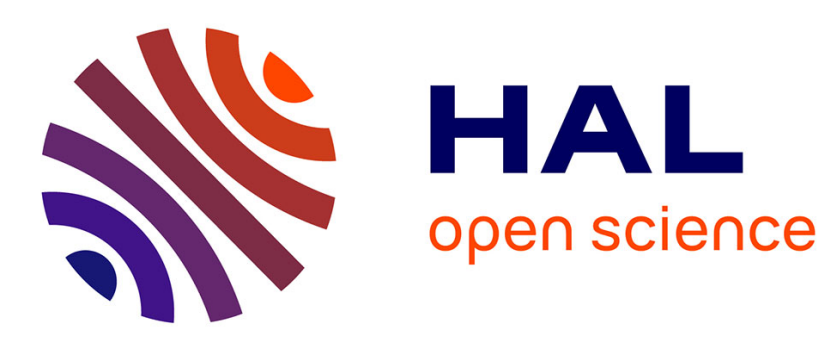

\title{
Fractional Poisson process: long-range dependence and applications in ruin theory
}

Romain Biard, Bruno Saussereau

\section{To cite this version:}

Romain Biard, Bruno Saussereau. Fractional Poisson process: long-range dependence and applications in ruin theory. Journal of Applied Probability, 2014, 51 (3), pp.1271 - 1272 . hal-00831074

\section{HAL Id: hal-00831074 \\ https://hal.science/hal-00831074}

Submitted on 6 Jun 2013

HAL is a multi-disciplinary open access archive for the deposit and dissemination of scientific research documents, whether they are published or not. The documents may come from teaching and research institutions in France or abroad, or from public or private research centers.
L'archive ouverte pluridisciplinaire HAL, est destinée au dépôt et à la diffusion de documents scientifiques de niveau recherche, publiés ou non, émanant des établissements d'enseignement et de recherche français ou étrangers, des laboratoires publics ou privés. 


\title{
Fractional Poisson process: long-range dependence and applications in ruin theory
}

\author{
Romain Biard and Bruno Saussereau
}

\author{
Laboratoire de mathématiques de Besançon, \\ UMR CNRS 6623, \\ 16 route de Gray, \\ 25030 Besançon, France. \\ e-mail: romain.biard@univ-fcomte.fr; bruno. saussereau@univ-fcomte.fr
}

\begin{abstract}
We study a renewal risk model in which the surplus process of the insurance company is modeled by a compound fractional Poisson process. We establish the long-range dependence property of this non-stationary process. Some results for the ruin probabilities are presented in various assumptions on the distribution of the claim sizes.
\end{abstract}

AMS 2000 subject classifications: Primary 60G22, 60G55, 91B30; secondary 60K05, 33E12. Keywords and phrases: Fractional Poisson process, renewal process, long-range dependence, ruin probability.

\section{Introduction}

An increasing interest has recently been paid to anomalous diffusion, i.e. processes whose variances increase in time in accordance with a power law $t^{\gamma}$ with $\gamma \neq 1$. This is effectively the case in seismology (see [MTN02]) where power-law function are used to model earthquake interarrival times. One refers to [BSM07] for other geophysical applications of such power law interarrival times.

One of the model of random processes that demonstrates such a phenomenon is investigated in [RS00] in which the authors were the first to introduce the so called fractional Poisson process. They replace, in the differential equations governing the Poisson process, the time derivative with a fractional one (see also [BO09, BO10, Jum01, Las03] for similar approaches). Another point of view, also proposed in [RS00], consists of considering the characterization of the Poisson process as a sum of independent non-negative random variables. We assume that these random variables have the MittagLeffler distribution instead of the exponential distribution. This is the so-called renewal approach that have been studied by [Mai96, MGS04]. This will be the point of view that we adopt in our article. Note, however, that it has been proved in [MNV11], that fractional Poisson process (defined as a renewal process) coincides with the fractal time Poisson process which is defined as a the time-changed usual Poisson process with the right continuous inverse of a standard $\mathrm{H}$-stable subordinator.

The power law that governs the time evolution of the variance is often related to the notion of long range dependence. The long range dependence is well defined for stationary processes and little seems to be known about the extension of the long range dependence to non stationary processes. Such an extension has been proposed in [HY97] and this will be the starting point of a new result concerning the fractional Poisson process. Indeed we establish the long-range dependence property of the fractional Poissonian noise (see Proposition 1 below). This is the main result of this work and it is the subject of Section 3. This property clearly justifies the use of fractional Poisson process in many concrete models and give some precisions about the intuitive approach using the power decay of the variance. For practical purposes, we mention that the simulations of waiting times and parameter estimation for the fractional Poisson process have been carried out in [CUW10, UCS08].

Our main motivation to study the fractional Poisson process is its application in actuarial sciences. This is strongly motivated by the above comments, especially if we want to model extreme events like 
earthquake or storm. The long range dependence of the fractional Poisson process, as well as the fact that the expectation of the interarrival, are other admissible arguments to consider the following model.

We shall work with the renewal risk model in which the surplus process of the insurance company is modelled by

$$
R_{t}=u+c t-\sum_{i=1}^{N_{H}(t)} X_{i}, \quad t \geq 0
$$

where $u$ is the initial capital, $c$ is the constant premium rate and the sequence of independent and identically distributed random variable $\left(X_{i}\right)_{i \geq 1}$ models the sizes of the successive claims. To our knowledge, there is only the work of [BM13] that deals with a fractional model for insurance. In their work, the authors established a large deviation principle for the fractional Poisson process and prove asymptotic results for the ruin probabilities of an insurance model like the one given by (1).

In the second part of our work, we shall make all our possible to give an overview of all the known results that may be applied to this context. Some of them are easy, but we strength the fact that our investigations are a first step toward the description of fractional Poisson models of surplus process. Let us briefly describe the properties we deal with.

In Section 4, we use the duality relation between our model and a compound Poisson model with arbitrary claim size distribution. This allows us to establish a closed-form formula for the density of the time to ruin when the claim sizes are exponentially distributed. The ruin probability in finite and infinite time are also studied.

The ruin probability in the context of heavy tailed size is the topic of Section 5. The properties that we establish are a consequence of the light tailed distribution of the fractional Poisson process.

At last, a Lundberg's inequality is proposed in Section 6 in which a bound for the ruin probability is proposed when the claim sizes have a light-tailed distribution.

Some preliminary results on the fractional Poisson process are gathered in Section 2 and the proof of a technical inequality is proposed in the Appendix A. We recall that Section 3 is devoted to the long-range dependence property.

\section{Preliminaries on the fractional Poisson process}

The fractional Poisson process was first defined in [RS00] as a renewal process with Mittag-Leffler waiting times. This means that it has independent, and identically distributed waiting times $\left(\Delta_{T_{k}}\right)_{k \geq 1}$, with distribution given by

$$
\mathbf{P}\left(\Delta_{T_{k}}>t\right)=E_{\mathrm{H}}\left(-\lambda t^{\mathrm{H}}\right)
$$

for $\lambda>0$ and $0<\mathrm{H} \leq 1$, where

$$
E_{\mathrm{H}}(z)=\sum_{k=0}^{\infty} \frac{z^{k}}{\Gamma(1+\mathrm{H} k)}
$$

is the Mittag-Leffler function ( $\Gamma$ denotes the Euler's Gamma function) which is defined for any complex number $z$. One can also characterized the distribution of the waiting times by their Laplace transform

$$
L_{H}(\xi)=\mathbf{E}\left(\exp \left(-\xi \Delta_{T_{k}}\right)\right)=\frac{\lambda}{\lambda+\xi^{\mathrm{H}}} .
$$

With $T_{n}=\Delta_{T_{1}}+\ldots+\Delta_{T_{n}}$ the time of the $n$th jump, the process $\left(N_{\mathrm{H}}(t)\right)_{t \geq 0}$ defined by

$$
N_{\mathrm{H}}(t)=\max \left\{n \geq 0: T_{n} \leq t\right\}=\sum_{k \geq 1} 1_{T_{k} \leq t}
$$

is a renewal process with Mittag-Leffler waiting times. It is called a fractional Poisson process of parameter $\mathrm{H}$. When necessary, we write $\Delta_{T_{k}}=T_{k}-T_{k-1}$ with the convention $T_{k-1}=0$ if $k=0$ (hence $\Delta_{T_{1}}=T_{1}$ ). Of course when $\mathrm{H}=1$, the Mittag-Leffler function with parameter 1 is the 
exponential function, and the waiting times become exponential. Thus a fractional Poisson process of parameter $\mathrm{H}=1$ is the usual Poisson process. As in the classical case, one remarks that we do not specify the dependence on $\lambda$ in the notation of the fractional Poisson process $N_{\mathrm{H}}$. This is done intentionally for reading convenience because the parameter $\lambda$ plays a minor role in the properties of the process.

In [RS00], it is proved that there exists a constant $C$ such that

$$
\mathbf{P}\left(\Delta_{T_{k}}>t\right) \sim_{t \rightarrow+\infty} C t^{-\mathrm{H}} .
$$

Consequently the inter-arrivals $\Delta_{T_{k}}$ have heavy tails and infinite mean for $0<\mathrm{H}<1$. We shall use in the sequel that the fractional Poisson process is light-tailed, that is $\mathbf{E}\left(e^{\xi N_{H}(t)}\right)<\infty$ for any $\xi>0$. This is a consequence of the existence of the moment generating function, whose expression is given by

$$
\mathbf{E}\left(z^{N_{\mathrm{H}}(t)}\right)=E_{\mathrm{H}}\left(\lambda t^{\mathrm{H}}(z-1)\right)
$$

for any $z>0$ (see [CUW10, Jum01, Las03]). The above result has been proved using fractional differential equations and fractional calculus. One notices that an alternative approach is feasible using the representation (see [MNV11]) of the fractional Poisson process as fractal time Poisson process $\left(N_{1}\left(E_{\mathrm{H}}(t)\right)\right)_{t>0}$. This process is defined as the time-changed usual Poisson process $\left(N_{1}(t)\right)_{t \geq 0}$ with $\left(E_{\mathrm{H}}(t)\right)_{t \geq 0}$ is the right continuous inverse of a standard $\mathrm{H}$-stable subordinator $\left(D_{\mathrm{H}}(t)\right)_{t \geq 0}$. This means that $E_{\mathrm{H}}(t)=\inf \left\{r>0: D_{\mathrm{H}}(r)>t\right\}$ where $\mathbf{E}\left[e^{-s D_{\mathrm{H}}(t)}\right]=\exp \left(-t s^{\mathrm{H}}\right)$. In the rest of our work, we will use the renewal approach. Nevertheless we think that it was worth mentioning the fractal time approach because it is a powerful tool that will be useful for obtaining new properties on both ruin problems and Poisson process studies. For example, one may deduce diffusion kind approximation of the risk process by $\mathrm{H}$-stable processes. This will be the subject of further investigations in future works.

\section{Long-range dependence}

The aim of this section is to prove that the fractional Poissonian noise $\left(X_{j}^{\mathrm{H}}\right)_{j \geq 1}$ defined for $j \geq 1$ by

$$
X_{j}^{\mathrm{H}}=N_{\mathrm{H}}(j)-N_{\mathrm{H}}(j-1),
$$

has a long-range dependence property. Long-range dependence is frequently understood in terms of power-law decay of the correlation function and it is standard in theory of stochastic processes to apply the notion of long range dependence only to stationary processes. It is also the case for a renewal processes $\left(N_{t}\right)_{t \geq 0}$ for which the notion of long-range dependence is defined as

$$
\limsup _{t \rightarrow \infty} \frac{\operatorname{Var}\left(N_{t}\right)}{t}=\infty
$$

when it is assumed that the process is stationary. It is known that the condition (5) is then equivalent to the infiniteness of the second moment of the inter-arrivals. This is the case for the inter-arrivals $\Delta_{T_{k}}$ of the process $N_{\mathrm{H}}$. This remark motivates that the fractional Poisson process is quickly said to have the long-range dependence property. One refers to [Dal99, DV97, DVJ03] for further informations about long range dependence for stationary point processes.

The stationarity assumption is not always fulfilled in certain areas of application and this is effectively the case in our study. So the above comments can not justify the long-range dependence property. Thus the aim of this section is to select the appropriate notion of long range dependence for non stationary processes. For that sake, it has been suggested in [HY97] to modify existing second-order definitions of long range dependence to apply to non stationary processes as well. Here is the definition proposed in the aforementioned work. 
Definition 1. A second order process $\left(X_{m}\right)_{m \geq 1}$ (not necessarily stationary) has the property of longrange dependence if the block mean process

$$
Y_{t}^{(m)}=\frac{\sum_{j=t m-m+1}^{j=t m} X_{j}}{\sum_{j=t m-m+1}^{j=t m} \operatorname{Var}\left(X_{j}\right)}
$$

defined for an integer $t \geq 1$ satisfies

$$
\lim _{m \rightarrow \infty}\left(\sum_{j=t m-m+1}^{j=t m} \operatorname{Var}\left(X_{j}\right)\right) \operatorname{Var}\left(Y_{t}^{(m)}\right)=+\infty .
$$

Remark. If the process $\left(X_{m}\right)_{m \geq 1}$ is centered, then it has the long range dependence property if

$$
\lim _{m \rightarrow \infty}\left(\sum_{j=t m-m+1}^{j=t m} \mathbf{E}\left(X_{j}^{2}\right)\right) \operatorname{Var}\left(Y_{t}^{(m)}\right)=+\infty
$$

with

$$
Y_{t}^{(m)}=\frac{\sum_{j=t m-m+1}^{j=t m} X_{j}}{\sum_{j=t m-m+1}^{j=t m} \mathbf{E}\left(X_{j}^{2}\right)} .
$$

Thus we have the formulation proposed in [HY97].

Remark. In the above definition, if the process $\left(X_{m}\right)_{m \geq 1}$ is centered and stationary, then (6) implies that

$$
\lim _{m \rightarrow \infty} \frac{\operatorname{Var}\left(\sum_{j=1}^{m} X_{j}\right)}{m}=\infty .
$$

Thus the variance of the sample mean of $m$ consecutive observations grows more slowly asymptotically than a sequence of independent identically distributed random variables. We recall that a sufficient condition for (7) is $\lim _{m \rightarrow \infty} \sum_{j=1}^{m} \operatorname{Cov}\left(X_{0}, X_{j}\right)=\infty$. Consequently, the formulation (6) is in accordance with the heuristic approaches and the usual definitions of the notion of long range dependence. One refers to the presentation of [HY97] for further discussion about this topic.

Using the notion of long-range dependence stated in Definition 1, we have the following proposition.

Theorem 1. The fractional Poissonian noise $\left(X_{j}^{\mathrm{H}}\right)_{j \geq 1}$ has the long-range dependence property for any $\mathrm{H} \in(0 ; 1)$.

Before proving this result we will use the following tools. Theorem 1 will be proved as soon as the convergence (6) will hold. So for a fixed integer $t \geq 1$, we denote

$$
\Delta_{t}^{(m)}=\frac{\operatorname{Var}\left(\sum_{j=t m-m+1}^{j=t m} X_{j}^{\mathrm{H}}\right)}{\sum_{j=t m-m+1}^{j=t m} \operatorname{Var}\left(X_{j}^{\mathrm{H}}\right)} .
$$

We remark that

$$
\Delta_{t}^{(m)}=\frac{\operatorname{Var}\left(N_{\mathrm{H}}(t m)-N_{\mathrm{H}}(t m-m)\right)}{\sum_{j=t m-m+1}^{j=t m} \operatorname{Var}\left(N_{\mathrm{H}}(j)-N_{\mathrm{H}}(j-1)\right)} .
$$

Therefore we need the variances of the increments of the fractional Poisson process. These quantities do not follow easily from the expression of the moment generating functions. Here we use the fact that the fractional Poisson process is a renewal process and we use the known result about the factorial moments of a renewal process. We have the following lemma. 
Lemma 2. Let $0 \leq s \leq t$, we have

$$
\mathbf{E}\left[\left(N_{\mathrm{H}}(t)-N_{\mathrm{H}}(s)\right) \times\left(N_{\mathrm{H}}(t)-N_{\mathrm{H}}(s)-1\right)\right]=2 \mathrm{H}\left(\frac{\lambda}{\Gamma(1+\mathrm{H})}\right)^{2} \int_{s}^{t}(t-r)^{\mathrm{H}} r^{\mathrm{H}-1} d r .
$$

Proof. We use the renewal function

$$
M_{\mathrm{H}}(t)=\mathbf{E}\left(N_{\mathrm{H}}(t)\right)=\frac{\lambda t^{\mathrm{H}}}{\Gamma(1+\mathrm{H})}
$$

and Proposition 1 in [Lag05] (see also [Dal99]).

Now we can prove Theorem 1.

Proof. For a fixed integer $t \geq 1$, we investigate the asymptotic behaviour of $\Delta_{t}^{(m)}$ defined in (8) as $m$ goes to infinity. By (9) and (10), we have

$$
\mathbf{E}\left(\left(N_{\mathrm{H}}(j)-N_{\mathrm{H}}(j-1)\right)^{2}\right)=2 \mathrm{H}\left(\frac{\lambda}{\Gamma(1+\mathrm{H})}\right)^{2} \int_{j-1}^{j}(j-r)^{\mathrm{H}} r^{\mathrm{H}-1} d r+\frac{\lambda}{\Gamma(1+\mathrm{H})}\left(j^{\mathrm{H}}-(j-1)^{\mathrm{H}}\right) .
$$

Since

$$
\int_{j-1}^{j}(j-r)^{\mathrm{H}} r^{\mathrm{H}-1} d r \leq \int_{j-1}^{j} r^{\mathrm{H}-1} d r=\frac{1}{\mathrm{H}}\left(j^{\mathrm{H}}-(j-1)^{\mathrm{H}}\right)
$$

we deduce that

$$
\begin{aligned}
\sum_{j=t m-m+1}^{j=t m} \mathbf{E}\left(\left(N_{\mathrm{H}}(j)-N_{\mathrm{H}}(j-1)\right)^{2}\right) & \leq \frac{\lambda}{\Gamma(1+\mathrm{H})}\left(\frac{2 \lambda}{\Gamma(1+\mathrm{H})}+1\right)\left[t^{\mathrm{H}}-(t-1)^{\mathrm{H}}\right] m^{\mathrm{H}} \\
& \leq t^{\mathrm{H}} \frac{\lambda}{\Gamma(1+\mathrm{H})}\left(\frac{2 \lambda}{\Gamma(1+\mathrm{H})}+1\right) m^{\mathrm{H}} .
\end{aligned}
$$

Using similar arguments, one has

$$
\begin{aligned}
\sum_{j=t m-m+1}^{j=t m}\left(\mathbf{E}\left(N_{\mathrm{H}}(j)-N_{\mathrm{H}}(j-1)\right)\right)^{2} & =\sum_{j=t m-m+1}^{j=t m}\left(\frac{\lambda}{\Gamma(1+\mathrm{H})} \mathrm{H} \int_{j-1}^{j} r^{\mathrm{H}-1} d r\right)^{2} \\
& \geq\left(\frac{\mathrm{H} \lambda}{\Gamma(1+\mathrm{H})}\right)^{2} \sum_{j=t m-m+1}^{j=t m} j^{2 \mathrm{H}-2} \\
& \geq\left(\frac{\mathrm{H} \lambda}{\Gamma(1+\mathrm{H})}\right)^{2} m \times(t m)^{2 \mathrm{H}-2} \\
& \geq\left(\frac{\mathrm{H} \lambda t^{\mathrm{H}-1}}{\Gamma(1+\mathrm{H})}\right)^{2} m^{2 \mathrm{H}-1} .
\end{aligned}
$$

and consequently there exists a constant $C_{t, \lambda, \mathrm{H}}$ such that the denominator of $\Delta_{t}^{(m)}$ satisfies

$$
\sum_{j=t m-m+1}^{j=t m} \operatorname{Var}\left(N_{\mathrm{H}}(j)-N_{\mathrm{H}}(j-1)\right) \leq t^{\mathrm{H}} \frac{\lambda}{\Gamma(1+\mathrm{H})}\left(\frac{2 \lambda}{\Gamma(1+\mathrm{H})}+1\right) \times m^{\mathrm{H}} \times\left(1-C_{t, \lambda, \mathrm{H}} m^{\mathrm{H}-1}\right) .
$$

In the same way, by (9) and (10), we also may write that

$$
\begin{aligned}
\operatorname{Var}\left(N_{\mathrm{H}}(t m)-N_{\mathrm{H}}(t m-m)\right) & =2 \mathrm{H}\left(\frac{\lambda}{\Gamma(1+\mathrm{H})}\right)^{2} \int_{t m-m}^{t m}(t m-r)^{\mathrm{H}} r^{\mathrm{H}-1} d r \\
& +\frac{\lambda}{\Gamma(1+\mathrm{H})}\left((t m)^{\mathrm{H}}-(t m-m)^{\mathrm{H}}\right)-\left\{\frac{\lambda}{\Gamma(1+\mathrm{H})}\left((t m)^{\mathrm{H}}-(t m-m)^{\mathrm{H}}\right)\right\}^{2} .
\end{aligned}
$$


Since

$$
\int_{t m-m}^{t m}(t m-r)^{\mathrm{H}} r^{\mathrm{H}-1} d r=(t m)^{2 \mathrm{H}} \int_{1-1 / t}^{1}(1-u)^{\mathrm{H}} u^{\mathrm{H}-1} d u \geq(t m)^{2 \mathrm{H}} \mathcal{B}(1+\mathrm{H}, \mathrm{H})
$$

where $\mathcal{B}$ denotes the beta function, defined for $a>0$ and $b>0$ by

$$
\mathcal{B}(a, b)=\int_{0}^{1} u^{a-1}(1-u)^{b-1} d u=\frac{\Gamma(a) \Gamma(b)}{\Gamma(a+b)},
$$

where we have used the notation $\Gamma$ for the gamma function. Thus we obtain

$$
\begin{aligned}
& \operatorname{Var}\left(N_{\mathrm{H}}(t m)-N_{\mathrm{H}}(t m-m)\right) \geq\left(\frac{\lambda}{\Gamma(1+\mathrm{H})}\right)^{2}\left[2 \mathrm{H} t^{2 \mathrm{H}} \mathcal{B}(1+\mathrm{H}, \mathrm{H})-\left\{t^{\mathrm{H}}-(t-1)^{\mathrm{H}}\right\}^{2}\right] m^{2 \mathrm{H}} \\
& +\frac{\lambda}{\Gamma(1+\mathrm{H})}\left(t^{\mathrm{H}}-(t-1)^{\mathrm{H}}\right) m^{\mathrm{H}} \\
& \geq\left(\frac{\lambda}{\Gamma(1+\mathrm{H})}\right)^{2} t^{2 \mathrm{H}}[2 \mathrm{H} \mathcal{B}(1+\mathrm{H}, \mathrm{H})-1] m^{2 \mathrm{H}} \\
& +\frac{\lambda}{\Gamma(1+\mathrm{H})}\left(t^{\mathrm{H}}-(t-1)^{\mathrm{H}}\right) m^{\mathrm{H}}
\end{aligned}
$$

where we have used the inequality $\left(t^{\mathrm{H}}-(t-1)^{\mathrm{H}}\right)^{2} \leq t^{2 \mathrm{H}}$. For $m$ sufficently big, we inject (13) and(14) into (8). This yields

$$
\begin{aligned}
\Delta_{t}^{(m)} & \geq\left\{t^{\mathrm{H}} \frac{\frac{\lambda}{\Gamma(1+\mathrm{H})}}{2 \frac{\lambda}{\Gamma(1+\mathrm{H})}+1}[2 \mathrm{H} \mathcal{B}(1+\mathrm{H}, \mathrm{H})-1] m^{\mathrm{H}}+\frac{1}{2 \frac{\lambda}{\Gamma(1+\mathrm{H})}+1}\right\} \frac{1}{1-C_{t, \lambda, \mathrm{H}} m^{\mathrm{H}-1}} \\
& \geq\left\{\frac{t^{\mathrm{H}} \lambda}{2 \lambda+\Gamma(1+\mathrm{H})}[2 \mathrm{H} \mathcal{B}(1+\mathrm{H}, \mathrm{H})-1] m^{\mathrm{H}}+\frac{\Gamma(1+\mathrm{H})}{2 \lambda+\Gamma(1+\mathrm{H})}\right\} \frac{1}{1-C_{t, \lambda, \mathrm{H}} m^{\mathrm{H}-1}} .
\end{aligned}
$$

We shall employ the following technical inequality

$$
2 \mathrm{H} \mathcal{B}(1+\mathrm{H}, \mathrm{H})-1>0
$$

which is valid for any $\mathrm{H} \in(0,1)$. Its proof is postponed in the Appendix A. Thus $\lim _{m \rightarrow \infty} \Delta_{t}^{(m)}=+\infty$ and consequently the long-range dependence property holds true.

\section{Probability of ruin with exponential claim sizes}

In this section, we restrict ourselves to the case of exponential claims. To be more precise, we consider the model

$$
R_{t}=u+c t-\sum_{i=1}^{N_{H}(t)} X_{i}, \quad t \geq 0
$$

where $u>0$ and the random variables $\left(X_{i}\right)_{i \geq 1}$ are non negative, independent and identically distributed as $\mathcal{E}(\mu)$ for $\mu>0$. It is also assumed that the sequence of claim sizes is independent of the fractional Poisson process $N_{\mathrm{H}}$. We point out the fact that the ruin problem is non trivial in infinite time for any $c>0$ because we have $\mathbf{E}\left(X_{1}-c T_{1}\right)=-\infty$.

\subsection{Closed-form representation for the distribution of the ruin time}

First of all, we derive an explicit formula for the distribution of the ruin time $\tau$ defined by

$$
\tau=\inf \left\{t>0: R_{t}<0\right\}
$$


This formula is a direct application of the main result stated in the paper of Borovkov and Dickson (see [BD08]) in which the study of the ruin time distribution for a Sparre Andersen process with exponential claim sizes is investigated.

Proposition 3. Under the above assumptions on the model described by Equation (16), the distribution of the ruin time $\tau$ has a density $p_{\tau}$ given by

$$
p_{\tau}(t)=e^{-\mu(u+c t)} \sum_{n=0}^{\infty} \frac{\mu^{n}(u+c t)^{n-1}}{n !}\left(u+\frac{c t}{n+1}\right) f_{\mathrm{H}}^{*(n+1)}(t)
$$

where $f_{\mathrm{H}}^{* n}$ denotes the $n-$ fold convolution of the function $f_{\mathrm{H}}$ defined by for $t \geq 0$ by

$$
f_{\mathrm{H}}(t)=u t^{\mathrm{H}-1} E_{\mathrm{H}, \mathrm{H}}\left(-\lambda t^{\mathrm{H}}\right)
$$

where

$$
E_{\alpha, \beta}(z)=\sum_{k=0}^{\infty} \frac{z^{k}}{\Gamma(\alpha k+\beta)}
$$

is the generalized two-parameter Mittag-Leffler function.

Proof. We apply Theorem 1 in [BD08] with the density $f_{\mathrm{H}}$ of the inter-arrival $\Delta_{T_{n}}$ whose expression is given in [CUW10].

\subsection{Ruin probability in finite time}

In this short subsection, we are interested in the expression of the Laplace transform of the probability $\psi(u, t)$ of ruin with finite time $0<t<\infty$ defined by

$$
\psi(u, t)=\mathbf{P}\left(R_{s}<0, \text { for some } s \leq t\right) .
$$

The result stated hereafter is again a straightforward application of Theorem 1 in [Mal98] (see also [WL02]). In this paper, the author uses a duality between classical risk process in which the aggregate claims up to time $t$ is modelled as a compound Poisson process and dual risk process in which the claim sizes are exponential and the inter-arrival times have another law. One refers to [MR04] for further precision about this duality. The Laplace transform of $\psi(u, t)$ is given in the following proposition.

Proposition 4. For any $x>0$ it holds that

$$
\xi \int_{0}^{\infty} e^{-\xi t} \psi(u, t) d t=1-y(\xi) \exp \{-u \mu(1-y(\xi))\}, \quad \xi>0
$$

where $y(\xi)$ is the unique solution of the equation

$$
y(\xi)=\frac{\lambda}{\lambda+(\xi+c \mu(1-y(\xi)))^{\mathrm{H}}}, \quad \xi>0 .
$$

Remark. It is well known that Equation (19) has a unique solution. Indeed, since we have denoted $\xi \mapsto L_{\mathrm{H}}(\xi)=\lambda /\left(\lambda+\xi^{\mathrm{H}}\right)$ the Laplace transform of the waiting times $\Delta_{T_{k}}$, solving Equation (19) is equivalent to the resolution of

$$
L_{\mathrm{H}}(\xi+C-C s)-s=0
$$

for a fixed $\xi>0$ with $C=c \mu$. Since the left hand side is a convex function with a negative value at $s=1$ and a positive value at $s=0$, it follows that there exists a unique root $y(\xi)$.

Of course, for practical purpose, the above proposition requires the numerical inversion of the Laplace transform. Numerical examples of such inversion will not be discussed here. 


\subsection{Ruin probability in infinite time}

In this subsection, we are interested in the infinite time ruin probability defined by

$$
\psi(u)=\mathbf{P}\left(R_{s}<0, \text { for some } s \geq 0\right) .
$$

Since the fractional Poisson process is a renewal process, we are in the framework of Theorem VI.2.2 in [AA10] because $\mu E\left[\Delta_{T_{1}}\right]=+\infty$.

Proposition 5. Under the assumptions of this section, we have

$$
\psi(u)=\left(1-\frac{\gamma}{\mu}\right) e^{-\gamma u},
$$

where $\gamma>0$ is the unique solution of

$$
\gamma^{\mathrm{H}}-\mu \gamma^{\mathrm{H}-1}+\frac{\lambda}{c^{\mathrm{H}}}=0 .
$$

Remark. Equation (20) can be explicitly solved for some $\mathrm{H} \in(0,1]$ (e.g. $\mathrm{H}=1 / 2,1 / 3$ or $2 / 3$ ) and easily worked out numerically in the general case. For $\mathrm{H}=1$, we retrieve the Poisson case $\gamma=\mu-\lambda / c$.

\section{Ruin probability in the presence of heavy tailed claim sizes}

In this section, we are concerned with distributions of the claim sizes $\left(X_{i}\right)_{i \geq 1}$ having a heavy right tail $\bar{F}(t)=1-F(t)$ with $F(t)=\mathbf{P}\left(X_{1} \leq t\right)$. Again, we consider the model

$$
R_{t}=u+c t-\sum_{i=1}^{N_{H}(t)} X_{i}, \quad t \geq 0
$$

and it is assumed that the distribution of $X_{1}$ is sub-exponential. We recall that $F$ is a sub-exponential distribution if it is concentrated on $(0, \infty)$ and if $\lim _{t \rightarrow \infty} \overline{F^{* 2}}(t) / \bar{F}(t)=2$ where $F^{* 2}$ is the convolution square. Since $X_{1}$ is now heavy tailed, its mean is not necessarily finite. Our result will state an equivalent of the probability

$$
\psi(u, t)=\mathbf{P}\left(R_{s}<0, \text { for some } s \leq t\right)
$$

of ruin at time $t$, as the initial capital $u$ tends to infinity. This will be a consequence of the behaviour of the tail of a random sum, when the random number of terms is light tailed and with sub-exponential heavy tailed independent random variables in the sum. This is stated in the next well-known Lemma which is exactly the Lemma X.2.2 in [AA10]. We recall it for the sake of conciseness.

Lemma 6. Let $\left(Y_{i}\right)_{i \geq 1}$ be a sequence of independent and identically distributed random variables with a common sub-exponential distribution $F$ and let $K$ be an independent integer-valued random variable satisfying $\mathbf{E}\left(z^{K}\right)<\infty$ for some $z>1$. Then it holds that

$$
\mathbf{P}\left(\sum_{i=1}^{K} Y_{i}>x\right) \sim \mathbf{E}(K) \bar{F}(x) \text { as } x \rightarrow \infty .
$$

Now we can state the following proposition.

Proposition 7. Let $\left(R_{t}\right)_{t \geq 0}$ be the risk process given by (21). If the distribution $F$ of the claim sizes is sub-exponential, then

$$
\psi(u, t) \sim \mathbf{E}\left(N_{\mathrm{H}}(t)\right) \bar{F}(u)
$$

as $u$ goes to $+\infty$. 
We remark that we also have

$$
\psi(u, t) \sim \frac{\lambda t^{\mathrm{H}} \bar{F}(u)}{\Gamma(1+\mathrm{H})} \text { as } u \rightarrow \infty,
$$

thanks to the explicit expression on the mean of $N_{\mathrm{H}}(t)$ given in (10).

Proof. We start from the inequalities

$$
\mathbf{P}\left(\sum_{i=1}^{N_{\mathrm{H}}(t)} X_{i}>u+c t\right) \leq \psi(u, t) \leq \mathbf{P}\left(\sum_{i=1}^{N_{\mathrm{H}}(t)} X_{i}>u\right)
$$

and we apply Lemma 22 to $\mathbf{P}\left(\sum_{i=1}^{N_{H}(t)} X_{i}>x\right)$ with $x=u$ or $x=u+c t$. By (6) we know that $\mathbf{E}\left(z^{N_{H}(t)}\right)$ is finite for any $z>1$ so by Lemma 22 we deduce that

$$
\mathbf{P}\left(\sum_{i=1}^{N_{\mathrm{H}}(t)} X_{i}>x\right) \underset{x \rightarrow \infty}{\sim} \mathbf{E}\left(N_{\mathrm{H}}(t)\right) \bar{F}(x) .
$$

Moreover it holds that $\bar{F}(u+t) \underset{u \rightarrow \infty}{\sim} \bar{F}(u)$. Therefore (25) yields (23).

An extension of the previous result can be achieved for a $k$-dimensional risk processes

$$
\mathbf{R}_{t}=\mathbf{u}+\mathbf{c} t-\sum_{i=1}^{N_{H}(t)} \mathbf{X}_{i}, \quad t \geq 0 .
$$

In (26), the process $\left(\mathbf{R}_{t}\right)_{t \geq 0}$ is defined by $\mathbf{R}_{t}=\left(R_{t}^{1}, \ldots, R_{t}^{k}\right)$, where the processes $R^{j}$ satisfies

$$
R_{t}^{j}=u^{j}+c^{j} t-\sum_{i=1}^{N_{\mathrm{H}}(t)} X_{i}^{j}, \quad t \geq 0, \quad 1 \leq j \leq k .
$$

Clearly we have denoted $\mathbf{u}=\left(u^{1}, \ldots, u^{k}\right)$ the initial capital vector, $\mathbf{c}=\left(c^{1}, \ldots, c^{k}\right)$ the premium intensity vector and the claim vectors $\mathbf{X}_{i}$ are equal to $\left(X_{i}^{1}, \ldots, X_{i}^{k}\right)$ for $i \geq 1$. The sequence $\left(\mathbf{X}_{n}\right)_{n \geq 1}$ is a sequence of independent and identically distributed random vectors with a joint distribution that satisfied

$$
F\left(x_{1}, \ldots, x_{k}\right)=\mathbf{P}\left(X^{1} \leq x_{1}, \ldots, X^{k} \leq x_{k}\right)=\prod_{j=1}^{k} \mathbf{P}\left(X^{j} \leq x_{k}\right):=\prod_{j=1}^{k} F_{j}\left(x_{j}\right)
$$

with obvious notations. Since the number of claims $N_{\mathrm{H}}(t)$ in the model $(26)$ is light tailed (remind that $\mathbf{E}\left(z^{N_{H}(t)}\right)<\infty$ for any $z>0$ ), one may copy line to line the arguments developed in the proof of Proposition 9.4 in [AA10] in order to state the following result.

Proposition 8. Assume that the distributions of the claim sizes $F_{j}$ are sub-exponential for $1 \leq j \leq k$. For an initial capital vector $\mathbf{u}$, we denote $\tau_{\max }(\mathbf{u})$ the first time when all the components of $\mathbf{R}$ are negative:

$$
\tau_{\max }(\mathbf{u})=\inf \left\{s>0: \max \left\{R_{s}^{1}, \ldots, R_{s}^{k}\right\}<0\right\}
$$

then, for any $t>0$, it holds that

$$
\mathbf{P}\left(\tau_{\max }(\mathbf{u}) \leq t\right) \sim \mathbf{E}\left[\left(N_{\mathrm{H}}(t)\right)^{k}\right] \prod_{j=1}^{k} \overline{F_{j}}\left(u_{j}\right)
$$

when $u_{j} \rightarrow \infty$ for any $1 \leq j \leq k$ 


\section{Ruin probability with light-tailed claim sizes}

Now, in the model (16), we assume that the common distribution of $X_{i}$ is light-tailed (hence $\mathbf{E}\left(e^{\xi X_{1}}\right)<$ $\infty$ for any $\xi>0)$. Since the fractional Poisson process has a light-tailed distribution, we are interested in the tails of random sums of a light-tailed number of light-tailed terms. Using large deviations for fractional process, asymptotic results for ruin probabilities of an insurance model with a fractional Poisson claim number process have been studied in [BM13]. Their result apply in our situation.

The aim of this section is to provide non asymptotic results in the same spirit of the celebrated Lundberg's inequality. We denote by $\left(S_{\mathrm{H}}(t)\right)_{t \geq 0}$ the compound fractional Poisson process which is naturally defined as

$$
S_{\mathrm{H}}(t)=\sum_{i=1}^{N_{\mathrm{H}}(t)} X_{i}
$$

The independence of the process $N_{\mathrm{H}}$ and the sequence $\left(X_{i}\right)_{i \geq 1}$ allows us to calculate the moment generating function of $S_{H}$. Indeed, it is proved in [Las03] that for any $\xi>0$

$$
\mathbf{E}\left[e^{\xi \mathcal{S}_{\mathrm{H}}(t)}\right]=E_{\mathrm{H}}\left(\lambda t^{\mathrm{H}}(g(\xi)-1)\right),
$$

where the function $g$ is the Laplace transform of the random variables $X_{i}$ defined by $g(\xi)=\mathbf{E}\left(e^{\xi X_{1}}\right)$ for any $\xi>0$. We have the following bound on the ruin probability in finite time.

Proposition 9. Let $\left(R_{t}\right)_{t \geq 0}$ be the risk process given by (21). Then for any $t>0$, there exists $\xi_{0}(t, \mathrm{H}, \lambda)$ such that for any $u \geq 0$ we have

$$
\psi(u, t) \leq 1.3 e^{-\xi_{0}(t, \mathrm{H}, \lambda) u}
$$

Remark. The constant $\xi_{0}(t, \mathrm{H}, \lambda)$ is explicitly given by (33). Of course (31) is meaningless unless

$$
u \geq u_{0}:=\ln (1.3) / \xi_{0}(t, \mathrm{H}, \lambda) \geq 1 /\left(4 \xi_{0}(t, \mathrm{H}, \lambda)\right) .
$$

Proof. By (25), we only have to estimate $\mathbf{P}\left(\sum_{i=1}^{N_{H}(t)} X_{i}>u\right)$. Using the Chebyshev exponential inequality and (30), we deduce that for any $\xi>0$,

$$
\mathbf{P}\left(\sum_{i=1}^{N_{\mathrm{H}}(t)} X_{i}>u\right)=\mathbf{P}\left(\exp \left(\xi S_{\mathrm{H}}(t)>e^{\xi u}\right) \leq e^{-\xi u} E_{\mathrm{H}}\left(\lambda t^{\mathrm{H}}(g(\xi)-1)\right) .\right.
$$

Now we prove an upper bound for the Mittag-Leffler function. We recall that

$$
E_{\mathrm{H}}(x)=\sum_{k=0}^{\infty} \frac{x^{k}}{\Gamma(1+\mathrm{H} k)} .
$$

The minimum value of $x \mapsto \Gamma(x)$ is achieved for positive values in a point denoted by $x_{0}$ (which is approximatively equal to 1.462$)$. We have $\Gamma\left(x_{0}\right) \simeq 0.8856$. Thus, if $g$ is the function defined for $\mathrm{H} \in(0,1)$ by $g(\mathrm{H})=\Gamma(1+\mathrm{H} k)$, we have $g^{\prime}(\mathrm{H})=k \Gamma^{\prime}(1+\mathrm{H} k)$. Hence $g$ has a minimum in $\mathrm{H}_{0}=\left(x_{0}-1\right) / k$. Thus

$$
g(H) \geq g\left(\frac{x_{0}-1}{k}\right) \geq \Gamma\left(1+\frac{x_{0}-1}{k} \times k\right)=\Gamma\left(x_{0}\right)
$$

and we obtain that for any $x \geq 0$,

$$
E_{\mathrm{H}}(x) \leq \frac{1}{\Gamma\left(x_{0}\right)} \sum_{k=0}^{\infty} x^{k}=\frac{1}{\Gamma\left(x_{0}\right)(1-x)}
$$


So we define $\xi_{0}(t, H, \lambda)$ as the unique positive real such that

$$
g\left(\xi_{0}\right)=1+\frac{2 \Gamma\left(x_{0}\right)-1}{\lambda t^{\mathrm{H}} \Gamma\left(x_{0}\right)} .
$$

We deduce from (32) that

$$
\mathbf{P}\left(\sum_{i=1}^{N_{\mathrm{H}}(t)} X_{i}>u\right) \leq \frac{e^{-\xi_{0}(t, \mathrm{H}, \lambda) u}}{\Gamma\left(x_{0}\right)\left(1-\lambda t^{\mathrm{H}}\left(g\left(\xi_{0}(t, \mathrm{H}, \lambda)\right)-1\right)\right)} \leq \frac{e^{-\xi_{0}(t, \mathrm{H}, \lambda) u}}{2 \Gamma\left(x_{0}\right)-1} \leq 1.3 \times e^{-\xi_{0}(t, \mathrm{H}, \lambda) u}
$$

and (31) is proved.

Remark. We notice that the estimation (32) is more accurate. For example, one may use it by plotting the function $\xi \mapsto e^{-\xi u} E_{\mathrm{H}}\left(\lambda t^{\mathrm{H}}(g(\xi)-1)\right)$ for small values of $\xi$ and check numerically the eventual minimum. Such procedure is feasible since the Mittag-Leffler function is now implemented on scientific software.

\section{Appendix A: Proof of Inequality (15)}

We denote $f$ the function defined by $x \mapsto \ln (2 x \mathcal{B}(1+x, x))$ for $x \in(0,1)$. We follow some technical trick used in [Sau12]. The inequality (15) will be a consequence of the positivity of $f$ on the interval $(0,1)$. Since $x \Gamma(x)=\Gamma(x+1)$, we have

$$
2 x \mathcal{B}(1+x, x)=\frac{2 x \Gamma(x) \Gamma(x+1)}{\Gamma(2 x+1)}=\frac{2 \Gamma(x+1)^{2}}{\Gamma(2 x+1)},
$$

and consequently

$$
f(x)=\ln (2)+2 \ln \Gamma(x+1)-\ln \Gamma(2 x+1) .
$$

We denote $\psi$ the function $(\ln \Gamma)^{\prime}=\Gamma^{\prime} / \Gamma$ (usually called the digamma function). We obtain that

$$
f^{\prime}(x)=2(\psi(x+1)-\psi(2 x+1)) .
$$

Since $\psi^{\prime}(x)=\sum_{k=0}^{\infty} \frac{1}{(x+k)^{2}}($ see [AAR99, page 13]), we deduce that

$$
\begin{aligned}
f^{\prime \prime}(x) & =2\left(\psi^{\prime}(x+1)-2 \psi^{\prime}(2 x+1)\right) \\
& =2 \sum_{k=0}^{\infty} \frac{1}{(x+1+k)^{2}}-\frac{1}{2(x+(k+1) / 2)^{2}} \\
& =\sum_{k=0}^{\infty} \frac{1}{(x+1+k)^{2}}+\sum_{k=0}^{\infty} \frac{1}{(x+1+k)^{2}}-\frac{1}{(x+(k+1) / 2)^{2}} \\
& =\sum_{k=0}^{\infty} \frac{1}{(x+1+k)^{2}}+\sum_{j=0}^{\infty}-\frac{1}{(x+(2 j+1) / 2)^{2}} \\
& =\sum_{k=0}^{\infty} \frac{1}{(x+1+k)^{2}}+\sum_{k=0}^{\infty}-\frac{1}{(x+k+1 / 2)^{2}} \\
& =\psi^{\prime}(x+1)-\psi^{\prime}(x+1 / 2) .
\end{aligned}
$$

Moreover, $\psi^{\prime}$ is a decreasing function because $\psi^{\prime \prime}(x)=-2 \sum_{k=0}^{\infty}(x+k)^{-3}$. It follows that $f^{\prime \prime} \leq 0$ on $(0,1)$. As a decreasing function, $f^{\prime}$ satisfies $f^{\prime}(x) \leq f^{\prime}(0)=0$ and consequently $f$ is itself a decreasing function. Finally, we deduce that

$$
f(x) \geq f(1)=\ln \left(\frac{2 \Gamma(2)^{2}}{\Gamma(3)}\right)=0,
$$

and the proof is complete. 


\section{References}

[AA10] S. Asmussen \& H. Albrecher - Ruin probabilities, second éd., Advanced Series on Statistical Science \& Applied Probability, 14, World Scientific Publishing Co. Pte. Ltd., Hackensack, NJ, 2010.

[AAR99] G. E. Andrews, R. Askey \& R. Roy - Special functions, Encyclopedia of Mathematics and its Applications, vol. 71, Cambridge University Press, Cambridge, 1999.

[BD08] K. A. Borovkov \& D. C. M. Dickson - On the ruin time distribution for a Sparre Andersen process with exponential claim sizes, Insurance Math. Econom. 42 (2008), no. 3, p. $1104-1108$.

[BM13] L. BEGHIN \& C. MACCI - Large deviations for fractional poisson processes, Statist. Probab. Lett. 83 (2013), p. 1193-1202.

[BO09] L. Beghin \& E. ORsingher - Fractional Poisson processes and related planar random motions, Electron. J. Probab. 14 (2009), p. no. 61, 1790-1827.

[BO10] - Poisson-type processes governed by fractional and higher-order recursive differential equations, Electron. J. Probab. 15 (2010), p. no. 22, 684-709.

[BSM07] D. A. Benson, R. Schumer \& M. M. Meerschaert - Recurrence of extreme events with power-law interarrival times, Geophysical Research Letters 34 (2007), no. 6, p. n/a-n/a.

[CUW10] D. O. CAhoy, V. V. UChaikin \& W. A. WoyCZYNSKI - Parameter estimation for fractional Poisson processes, J. Statist. Plann. Inference 140 (2010), no. 11, p. 3106-3120.

[Dal99] D. J. DALEY - The Hurst index of long-range dependent renewal processes, Ann. Probab. 27 (1999), no. 4, p. 2035-2041.

[DV97] D. J. DALEY \& R. VESILO - Long range dependence of point processes, with queueing examples, Stochastic Process. Appl. 70 (1997), no. 2, p. 265-282.

[DVJ03] D. J. DAley \& D. Vere-Jones - An introduction to the theory of point processes. Vol. I, second éd., Probability and its Applications (New York), Springer-Verlag, New York, 2003, Elementary theory and methods.

[HY97] C. C. Heyde \& Y. YAng - On defining long-range dependence, J. Appl. Probab. 34 (1997), no. 4, p. 939-944.

[Jum01] G. JUMARIE - Fractional master equation: non-standard analysis and Liouville-Riemann derivative, Chaos Solitons Fractals 12 (2001), no. 13, p. 2577-2587.

[Lag05] A. N. LAGERAS - A renewal-process-type expression for the moments of inverse subordinators, J. Appl. Probab. 42 (2005), no. 4, p. 1134-1144.

[Las03] N. Laskin - Fractional Poisson process, Commun. Nonlinear Sci. Numer. Simul. 8 (2003), no. 3-4, p. 201-213, Chaotic transport and complexity in classical and quantum dynamics.

[Mai96] F. MAINARDI - The fundamental solutions for the fractional diffusion-wave equation, Appl. Math. Lett. 9 (1996), no. 6, p. 23-28.

[Mal98] V. K. MaLinovski - Non-Poissonian claims' arrivals and calculation of the probability of ruin, Insurance Math. Econom. 22 (1998), no. 2, p. 123-138.

[MGS04] F. Mainardi, R. Gorenflo \& E. Scalas - A fractional generalization of the Poisson processes, Vietnam J. Math. 32 (2004), no. Special Issue, p. 53-64.

[MnV11] M. M. Meerschaert, E. Nane \& P. Vellaisamy - The fractional Poisson process and the inverse stable subordinator, Electron. J. Probab. 16 (2011), p. no. 59, 1600-1620.

[MR04] C. MAzZA \& D. RULliÈRE - A link between wave governed random motions and ruin processes, Insurance Math. Econom. 35 (2004), no. 2, p. 205-222.

[MTN02] R. M. W. Musson, T. Tsapanos \& C. T. NAKAS - A power-law function for earthquake interarrival time and magnitude, Bull. Seismol. Soc. Am. 92 (2002), no. 5, p. 1783-1794.

[RS00] O. N. RePIn \& A. I. SAICHev - Fractional Poisson law, Radiophys. and Quantum Electronics 43 (2000), no. 9, p. 738-741 (2001).

[Sau12] B. SAUSSEREAU - Transportation inequalities for stochastic differential equations driven by a fractional Brownian motion, Bernoulli 18 (2012), no. 1, p. 1-23.

[UCS08] V. V. Uchaikin, D. O. CAhoy \& R. T. Sibatov - Fractional processes: from Poisson to branching one, Internat. J. Bifur. Chaos Appl. Sci. Engrg. 18 (2008), no. 9, p. 2717-2725. 
R. Biard and B. Saussereau/Fractional Poisson process: long-range dependence and applications in ruin theory 13

[WL02] R. WANG \& H. LIU - On the ruin probability under a class of risk processes, Astin Bull. 32 (2002), no. 1, p. 81-90. 